\title{
INFLUÊNCIA DA BIOMASSA INICIAL SOBRE O CRESCIMENTO E A PRODUTIVIDADE DE PEIXES EM SISTEMA DE POLICULTIVO
}

\author{
Effects of initial biomass on fish growth and fishery productivity in polyculture systems
}

\author{
Zélia Maria Pimentel Nunes ${ }^{1}$, Xavier Lazzaro ${ }^{2}$, Alberto Carvalho Peret ${ }^{3}$
}

\begin{abstract}
RESUMO
Analisou-se o crescimento dos peixes, a composição das espécies e a produtividade de quatro policultivos (P75, P78, P87 e P207), visando melhorar o manejo e a produtividade pesqueira dos pequenos açudes $(0,1-5,0 \mathrm{ha})$ do SemiÁrido brasileiro. Simulou-se as condições desses açudes em viveiros com 120 e $5.000 \mathrm{~m}^{2}$ de área, sem renovação de água, utilizando moderada quantidade de adubo e fertilizante. A biomassa inicial variou de 75 a $207 \mathrm{~kg} \mathrm{ha}^{-1}$, sendo formada por: tilápia do Nilo (Oreochromis niloticus), curimatã pacu (Prochilodus argenteus), carpa comum (Cyprinus carpio), tambaqui (Colossoma macropomum) e tucunaré (Cichla ocellaris). Os peixes apresentaram baixo crescimento $\left(<0,01 \mathrm{~g} \mathrm{~g}^{-1} \mathrm{~d}^{-1}\right)$ após 75 dias de criação (P78 e 87). O crescimento do tambaqui, da tilápia e da curimatã foi reduzido após 53 dias (P75). Em moderada biomassa, o crescimento do tambaqui foi inferior ao da carpa e da curimatã (P207). A produtividade da tilápia atingiu $720 \mathrm{~kg} \mathrm{ha}^{-1} \mathrm{ano}^{-1}$ (P78), sendo reduzida para $220 \mathrm{~kg} \mathrm{ha}^{-1} \mathrm{ano}^{-1}$ devido ao processo reprodutivo (P75 e P207). A produtividade da carpa de $1.600 \mathrm{~kg} \mathrm{ha}^{-1} \mathrm{ano}^{-1}$ foi superior a dos outros peixes (P87). A biomassa inicial de $75 \mathrm{~kg}^{-1}$ (60:30:4:3:3\% de tilápia, tambaqui, carpa, curimatã e tucunaré, respectivamente) otimizou o crescimento e a produtividade dos peixes. A utilização de tilápias monossexadas e o fornecimento da alimentação suplementar ao tambaqui tornam-se imprescindíveis ao policultivo.
\end{abstract}

Termos para indexação: Policultivo, pequenos açudes, produtividade pesqueira.

\begin{abstract}
The analysed species composition, fish growth, and productivity of four polycultures (P75, P78, P87, and P207) with the objective to improve the small reservoir (0.1-5 ha) fishery management and productivity in the Brazilian semiarid region were conducted a experiment. To mimic reservoir conditions, we used 120 and $5,000 \mathrm{~m}^{2}$ ponds and evaporation and infiltration water loss was replaced. In addition, manure and fertilizers were used only moderately. The initial biomass of Nile tilapia Oreochromis niloticus, 'tambaqui' Colossoma macropomum, 'curimatã pacu' Prochilodus argenteus, common carp Cyprinus carpio, and 'tucunaré' Cichla ocellaris ranged from 75 to $207 \mathrm{~kg} \mathrm{ha}^{-1}$. The fish showed low growth rates $\left(<0.01 \mathrm{~g} \mathrm{~g}^{-1} \mathrm{~d}^{-1}\right)$ after 75 days of culture (P78 and P87). Tambaqui, tilapia, and curimatã growth decreased after 53 days (P75). In moderate biomass, tambaqui grew less than carp and curimatã did (P207). Tilapia productivity reached $720 \mathrm{~kg} \mathrm{ha}^{-1} \mathrm{yr}^{-1}$ (P78) and fell to $220 \mathrm{~kg}$ ha ${ }^{1} \mathrm{yr}^{-1}$ because of the reproductive process (P75 and P207). The carp productivity of $1,600 \mathrm{~kg} \cdot \mathrm{ha}^{-1} \mathrm{yr}^{-1}$ was higher than those of the other fish (P87). The $75 \mathrm{~kg} \cdot \mathrm{ha}^{-1}$ biomass level (60:30:4:3:3\% of tilapia, tambaqui, carp, curimatã, and tucunaré, respectively) optimized fish growth and productivity. The use of monosexed tilapia and tambaqui feed supplementation was indispensable for the success of polyculture.
\end{abstract}

Index terms: Polyculture, small reservoirs, fishery productivity.

(Recebido para publicação em 5 de maio de 2004 e aprovado em 23 de janeiro de 2006)

\section{INTRODUÇÃO}

O policultivo consiste na criação de organismos que ocupam diferentes nichos ecológicos num mesmo ambiente proporcionando um melhor aproveitamento dos recursos disponíveis, da área e, conseqüentemente, o aumento de produtividade (MILSTEIN, 1990). A combinação de diferentes espécies de peixes pode contribuir para melhorar as condições ambientais pelo aumento na disponibilidade de recursos alimentares e da oxigenação da água. A compreensão destas relações torna-se uma ferramenta essencial ao manejo do policultivo e a maximização da produção de peixes (MILSTEIN, 1997).

Woynarovich (1985) fundamenta o policultivo na composição das espécies, no ciclo de produção da matéria orgânica no viveiro, na produtividade natural e nas principais etapas do metabolismo aquático. A densidade de cada espécie está baseada na melhor utilização do alimento e proporcional à alimentação suplementar oferecida. Deve-se utilizar espécies com diferentes hábitos alimentares, mesma faixa de tolerância à temperatura, com idade, tamanho e crescimento similares para cada espécie.

'Engenheira de Pesca, DSc., Professor Adjunto da UFPA - Núcleo de Estudos Costeiros-Alameda Leandro Ribeiro, Aldeia - Campus Universitário de Bragança - 68.600-000 - Bragança, PA.

${ }^{2}$ Hidrobiólogo, PhD., Pesquisador do Instiut de Reserche pour le Developpment - IRD/França - UR098-FLAG, Centre IRD, Bel Air, BP 1386, Dakar, Senegal- lazzaro@ird.sn

${ }^{3}$ Biólogo, DSc., Professor Adjunto da Universidade Federal de São Carlos/UFSCar - Centro de Ciências biológicas e da Saúde - Departamento de Hidrobiologia - Via Washington Luiz km 235, Monjolinho - 13.565-905 - São Carlos, SP. 
Os pequenos açudes (0,1-5,0 ha) espalhados na paisagem semi-árida do Nordeste brasileiro têm múltiplo uso, mas ainda estão sendo subutilizados. A piscicultura semi-intensiva surge como uma alternativa ao melhor aproveitamento destes ecossistemas.

Segundo Molle \& Cadier (1992), no Semi-Árido, as estações seca e chuvosa condicionam o peixamento e a depesca; a disponibilidade de adubo reduz os gastos com alimentação e a utilização de alevinos grandes com maior capacidade natatória, de 6 a $10 \mathrm{~cm}$, reduz a predação por peixes carnívoros e diminui o tempo de criação. O tempo de criação restringe-se ao período de retenção de água pelo açude; o policultivo de peixes pode otimizar o uso dos açudes nos locais onde existem outras fontes de água para abastecimento humano.

Neste trabalho, analisou-se o crescimento dos peixes, a composição das espécies e a produtividade de quatro policultivos a fim de melhorar as técnicas de manejo e a produtividade de pequenos reservatórios. Para tanto, foram simuladas as condições de pequenos açudes em viveiros sem renovação de água, empregando-se quantidades moderadas de adubos e fertilizantes considerando o multiuso dos açudes.

\section{MATERIAL E MÉTODOS}

As biomassas iniciais dos policultivos analisados formados por: tilápia Oreochromis niloticus, curimatã Prochilodus argenteus e carpa Cyprinus carpio foi de78 $\mathrm{kg} \mathrm{ha}^{-1}$ (P78); o de tambaqui Colossoma macropomum, curimatã e carpa foi de $87 \mathrm{~kg} \mathrm{ha}^{-1}$ (P87). Esses policultivos (P78 e P87) foram realizados na 'Sun West Aquafarms' Ltda em Petrolina - PE. Foram utilizados seis viveiros semiescavados em terreno natural, com área individual de $120 \mathrm{~m}^{2}$ e profundidade média de $80 \mathrm{~cm}$. Cada policultivo constou de três repetições. O tempo de criação teve duração média de 90 dias, de agosto a novembro de 1998 (Tabela 1).

Analisou-se outros policultivos com dois níveis de biomassa inicial 75 (P75) e $207 \mathrm{~kg} \mathrm{ha}^{-1}$ (P207), formados por tilápia do Nilo, tambaqui, carpa comum e curimatã pacu, descritos na Tabela 1 , com duas repetições. Utilizou-se quatro viveiros de $5000 \mathrm{~m}^{2}$, da Unidade de Piscicultura de Bebedouro pertencente à Companhia de Desenvolvimento do Vale do São Francisco (PetrolinaPE). O tempo de criação teve duração média de 120 dias, janeiro a maio de 2000. Após a primeira amostragem (16/ $02 / 00)$, ao se verificar desovas de tilápia, uma baixa biomassa $\left(<2 \mathrm{~kg} \mathrm{ha}^{-1}\right)$ de tucunaré Chicla ocellaris foi introduzida nos viveiros.
A preparação dos viveiros constou da limpeza, exposição ao sol durante uma semana e a utilização de calcário dolomítico na proporção $100 \mathrm{~g} \mathrm{~m}^{-2}$ (P78 e P87) e $60 \mathrm{~g} \mathrm{~m}^{-2}$ (P75 e P207). Em seguida, os viveiros foram abastecidos e, dois dias após o abastecimento, foi feita a adubação orgânica com cama de galinha, utilizando-se $250 \mathrm{~g} \mathrm{~m}^{-2} \mathrm{e}$ uma fertilização nitrogenada, com uréia, numa concentração de $0,94 \mathrm{~g} \mathrm{~N} \mathrm{~m}^{-3}$ (P78 e P87). Nos viveiros dos P75 e P207, após o abastecimento de $60 \%$ do volume total dos viveiros, foi feita a adubação, utilizando-se 180 $\mathrm{g} \mathrm{m}^{-2}$ de esterco misto (bovinos e caprinos). No dia posterior, à adubação orgânica foi feita a fertilização na concentração de $0,8 \mathrm{~g} \mathrm{~N} \mathrm{~m}^{-3}$, fornecida pela uréia. Em seguida, procedeu-se o completo abastecimento dos viveiros.

Quinzenalmente foi feito o monitoramento das variáveis físicas, químicas e biológicas da água (NUNES, 2000). Após as análises da água dos viveiros, quando necessária, foi feita uma adubação complementar com cama de galinha na proporção de $125 \mathrm{~g} \mathrm{~m}^{-2} \mathrm{e}$, no dia seguinte, uma fertilização nitrogenada de 0,35 a $0,47 \mathrm{gN} \mathrm{m}^{-3}$. Ao se constatar valores de alcalinidade e dureza inferiores a 20 mg CaCO $\mathrm{L}^{-1}$ foram feitas calagens complementares com calcário dolomítico em taxas de 25 a $50 \mathrm{~g} \mathrm{~m}^{-2}$ para melhorar a capacidade de tamponamento da água. Devido à grande área dos viveiros (P75 e P207), fez-se necessário adicionar maiores quantidades de esterco. Nesse caso, a quantidade total de adubo a ser introduzida nos viveiros foi dividida em duas partes, sendo a primeira colocada logo após a realização das análises e, a segunda, oito dias após. O mesmo procedimento foi adotado para a fertilização nitrogenada.

O crescimento dos peixes foi acompanhado nas amostragens quinzenais (P78 e P87) e mensais (P75 e P207) feitas com rede de arrasto. Durante a biometria, obteve-se o peso médio dos indivíduos de cada espécie, utilizando-se uma balança digital (precisão \pm $5 \mathrm{~g}$ ). O comprimento total dos indivíduos foi medido com um ictiômetro $( \pm 1 \mathrm{~mm})$. Na análise do crescimento em peso utilizou-se a taxa de crescimento intrínseco (TCI):

$$
T C I=\frac{\left(W_{T+1}-W_{T}\right)}{T W_{T}}
$$

Sendo: $W_{T}=$ peso total médio no instante $\mathrm{T}$;

$W_{T+1}=$ peso total médio no instante subseqüente ao $\mathrm{T}$; $\mathrm{T}=$ intervalo de tempo. 
TABELA 1 - Estrutura e composição dos policultivos analisados.

\begin{tabular}{|c|c|c|c|c|c|c|}
\hline Peixes & Tratamento & $\begin{array}{l}\text { Densidade } \\
\left(\text { peixe.ha }^{-1}\right)\end{array}$ & $\begin{array}{l}\mathbf{W}_{\mathbf{T}} \\
(\mathrm{g})\end{array}$ & $\begin{array}{l}\mathbf{L}_{\mathrm{T}} \\
(\mathbf{c m})\end{array}$ & $\begin{array}{c}\text { Biomassa } \\
\text { Inicial }\left(\mathrm{kgha}^{-\mathbf{1}}\right)\end{array}$ & $\begin{array}{c}\text { Composição } \\
(\%)\end{array}$ \\
\hline Tilápia do Nilo & P78 & 6250 & $4,5 \pm 1,4$ & $6,4 \pm 0,6$ & $28,3 \pm 8,6$ & 36 \\
\hline Carpa comum & P78 & 3083 & $4,1 \pm 2,8$ & $6,4 \pm 1,6$ & $12,6 \pm 8,5$ & 16 \\
\hline Curimatã pacu & P78 & 1833 & $20,2 \pm 0,4$ & 12,3 & $37,0 \pm 0,7$ & 48 \\
\hline Total/ viveiro & P78 & 11.166 & & & 78 & 100 \\
\hline Tambaqui & P87 & 6250 & $3,6 \pm 0,3$ & $6,7 \pm 0,1$ & $22,2 \pm 1,5$ & 25 \\
\hline Carpa comum & P87 & 3083 & $7,7 \pm 4,9$ & $7,3 \pm 1,4$ & $24,0 \pm 15,2$ & 28 \\
\hline Curimatã pacu & P87 & 1833 & $22,0 \pm 3,9$ & $12,7 \pm 0,7$ & $40,3 \pm 7,0$ & 47 \\
\hline Total/ viveiro & P87 & 11.166 & & & 87 & 100 \\
\hline Tilápia do Nilo 1 & P75 & 1250 & $\begin{array}{l}17,9 \\
63,5\end{array}$ & $\begin{array}{l}10,22 \\
12,85\end{array}$ & 46,1 & 61 \\
\hline Tambaqui 1 & P75 & $800+565$ & 16,6 & 10,22 & 220 & 20 \\
\hline Tambaqui 2 & P/D & $800 \pm 565$ & 63 & 15,51 & 22,0 & 29 \\
\hline Carpa comum & P75 & 400 & 6,2 & 4,71 & 2,4 & 3 \\
\hline Curimatã pacu & P75 & 700 & 5,3 & 5,48 & 3,0 & 4 \\
\hline Tucunaré & P75 & 8 & 239,3 & - & 1,9 & $<3$ \\
\hline Total/ viveiro & P75 & 3.158 & & & 75 & 100 \\
\hline Tilápia do Nilo 1 & P207 & 4500 & 24,6 & 11,36 & 1341 & 65 \\
\hline Tilápia do Nilo 2 & 1201 & & 39,8 & 13,32 & & (0) \\
\hline Tambaqui 1 & P207 & 2450 & 15,6 & 10,22 & 66,0 & 32 \\
\hline Tambaqui 2 & 1201 & 2450 & 62,2 & 14,16 & 00,0 & 32 \\
\hline Carpa comum & P207 & 350 & 6,2 & 4,71 & 2,2 & 1 \\
\hline Curimatã pacu & P207 & 1300 & 5,3 & 5,48 & 3,0 & 2 \\
\hline Tucunaré & P207 & 7 & 264 & - & 1,8 & $\leq 1$ \\
\hline Total/ viveiro & P207 & 8.607 & & & 207 & 100 \\
\hline
\end{tabular}

$\mathrm{W}_{\mathrm{T}}=$ peso total.

$\mathrm{L}_{\mathrm{T}}=$ comprimento total.

As análises univariadas de variância (ANOVA's) foram empregadas para testar o efeito do tratamento na taxa de crescimento intrínseco e na produção de cada espécie de peixe entre os tratamentos P75 e P207. Também, foram realizadas ANOVA's para testar o efeito do tratamento na produção de curimatã, de carpa e na total dos policultivos P78 e P87.

\section{RESULTADOS E DISCUSSÃO}

A mortalidade ficou restrita à fase inicial de criação devido ao estresse causado pelo transporte e pelo manejo realizado durante a estocagem dos peixes. No policultivo
P87, o tambaqui apresentou elevada mortalidade o que resultou em uma baixa sobrevivência (28\%) e numa baixa produção. Essa situação foi observada para curimatã (P75 e P207). A tilápia apresentou baixa sobrevivência, com média de 49\% nos P75 e P207 (Tabela 2).

$\mathrm{Na}$ Figura 1 pode-se observar que os peixes apresentaram crescimento reduzido $\left(<0,01 \mathrm{~g} \mathrm{~g} \mathrm{~g}^{-1} \mathrm{~d}^{-1}\right)$ a partir de 75 dias de criação (P78 e P87). O crescimento inicial da tilápia foi de $0,147 \mathrm{~g} \mathrm{~g}^{-1} \mathrm{~d}^{-1}$ (P78) e $0,030 \mathrm{~g} \mathrm{~g}^{-1} \mathrm{~d}^{-1}(\mathrm{P} 75)$. No P207, esse peixe apresentou crescimento lento $\left(<0,020 \mathrm{~g} \mathrm{~g}^{-1} \mathrm{~d}^{-1}\right)$. No P75, as taxas de crescimento de tambaqui, tilápia e curimatã tornaram-se reduzidas após os 53 dias iniciais. 
TABELA 2 - Produtividade $\left(\mathrm{kg} \cdot \mathrm{ha}^{-1} \mathrm{ano}^{-1}\right)$ e sobrevivência de cada espécie nos policultivos.

\begin{tabular}{lrrrrrrrr}
\hline \multirow{2}{*}{ Espécie } & \multicolumn{3}{c}{ Produtividade $\left(\mathbf{k g ~ h a}^{-\mathbf{1}} \mathbf{a n o}^{-\mathbf{1}}\right)$} & \multicolumn{4}{c}{ Sobrevivência (\%) } \\
\cline { 2 - 9 } & $\mathbf{P 7 5}$ & $\mathbf{P ~ 7 8}$ & $\mathbf{P ~ 8 7}$ & $\mathbf{P ~ 2 0 7}$ & $\mathbf{P 7 5}$ & $\mathbf{P ~ 7 8}$ & P 87 & P 207 \\
\hline Tilápia do Nilo & 124,0 & 721,9 & - & 219,9 & 49,5 & 90,3 & - & 48,7 \\
Tambaqui & 331,6 & - & 256,7 & 117,4 & 89,2 & - & 28,0 & 92,2 \\
Carpa comum & 573,5 & 1524,9 & $1.606,8$ & 231,3 & 60,4 & 73,8 & 86,1 & 81,2 \\
Curimatã pacu & 31,6 & 176,0 & 267,0 & 36,6 & 22,1 & 92,2 & 100,0 & 24,6 \\
Tucunaré & 5,7 & - & - & 2,7 & 89,0 & - & - & 50,0 \\
\hline Total & $1.066,4$ & $2.422,8$ & $2.230,5$ & 607,9 & & & & \\
\hline
\end{tabular}
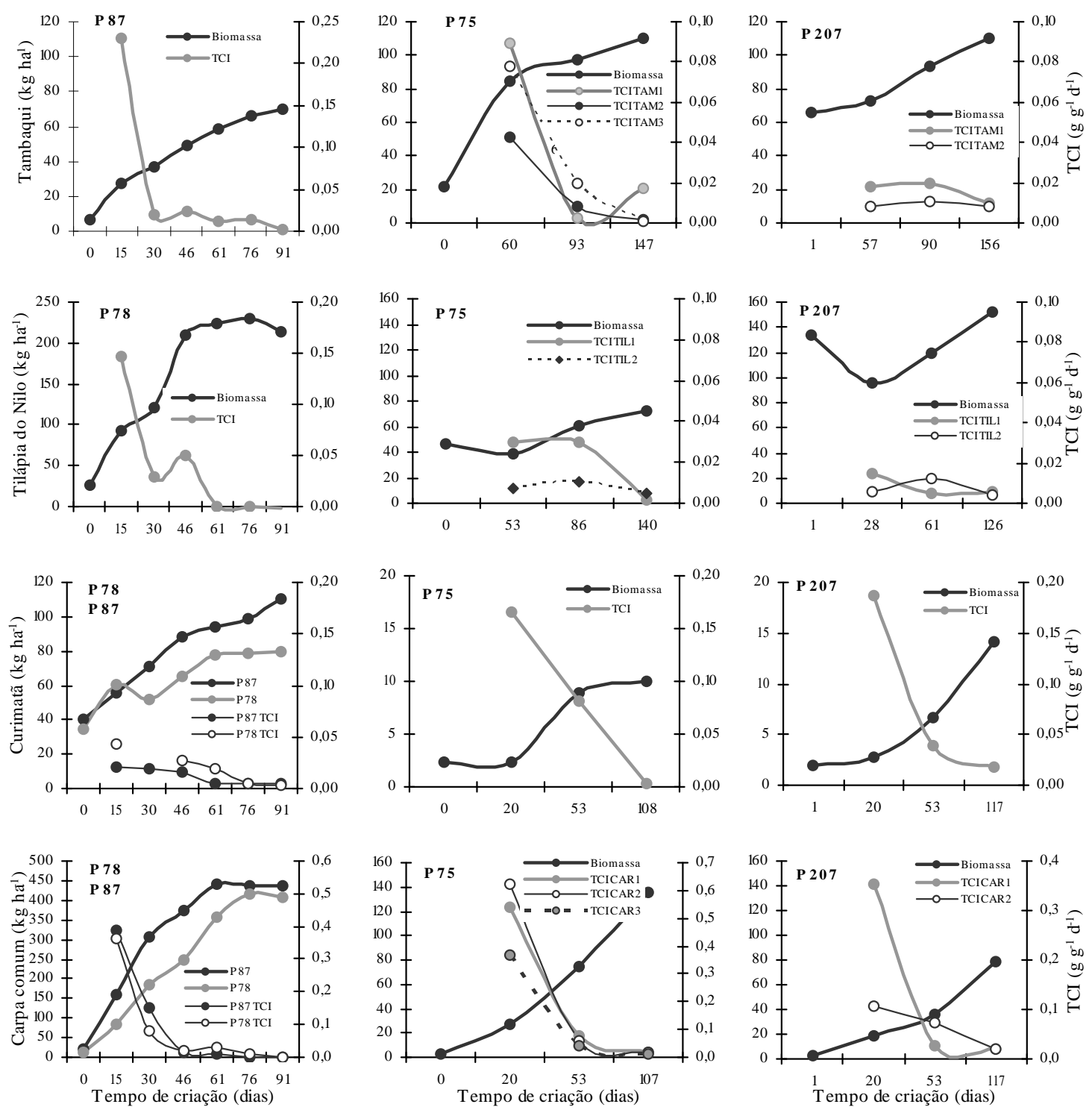

FIGURA 1 - Influência da biomassa sobre o crescimento das espécies.TC I= taxa de crescimento intrínseco.

Ciênc. agrotec., Lavras, v. 30, n. 6, p. 1083-1090, nov./dez., 2006 
No policultivo P87, o crescimento do tambaqui variou de 0,230 a $0,013 \mathrm{~g} \mathrm{~g}^{-1} \mathrm{~d}^{-1}$ nos primeiros setenta e cinco dias de criação. No P75, essa variação foi de 0,089 a $0,042 \mathrm{~g} \mathrm{~g}^{-1} \mathrm{~d}^{-1}$, nos primeiros 60 dias. $\mathrm{O}$ tambaqui teve o seu crescimento reduzido ao ser criado em moderada biomassa P207 ( $\mathrm{p}<0,05)$. O alimento natural de origem endógena foi limitante ao crescimento dos peixes em policultivo com moderada biomassa inicial. A limitação nutricional ficou bem caracterizada na heterogeneidade de tamanhos apresentada pelo tambaqui e na baixa porcentagem de peixes que atingiram o tamanho comercial (1\%). A melhor produtividade do tambaqui foi em viveiros com baixa biomassa inicial. O efeito da densidade sobre o crescimento e a necessidade do alimento suplementar para melhorar o desempenho do tambaqui foi observado por Nunes (2000) e corroborado pelos trabalhos de Moncayo \& Hernandez (1988) e Teichert-Coddington \& Green (1997).

Nos policultivos analisados, o alimento natural disponível nos viveiros (zooplâncton, insetos, gastrópodes, algas filamentosas) não foi suficiente para atender aos requerimentos nutricionais do tambaqui no que se refere à demanda por carboidratos (fonte de energia) entre os 60 e 90 dias de criação. Nesse período, o alimento natural com elevado teor protéico tornou a relação proteína: energia deficitária. Lima \& Goulding (1998) concluíram que os principais alimentos deste peixe são sementes e zooplâncton, que contribuem com 60 e $40 \%$ respectivamente, do suprimento energético. A maioria das sementes e frutos é composta por carboidratos, principal nutriente da dieta ingerida pelo tambaqui jovem na natureza.

$\mathrm{O}$ efeito da alimentação suplementar associado à adubação orgânica foi evidenciado por TeichertCoddington \& Green (1997) em policultivos de tilápia do Nilo e tambaqui. Nos viveiros, onde além da adubação orgânica (esterco de galinha) foi fornecida uma alimentação suplementar (25\% PB), o crescimento do tambaqui foi $422 \%$ superior ao obtido em viveiros adubados. Nessas mesmas condições, a tilápia apresentou crescimento $39 \%$ superior ao obtido em viveiros que foram somente adubados.

O crescimento da curimatã pode ser observado na Figura 1. No P78, a TCI variou de $0,043 \mathrm{~g} \mathrm{~g}^{-1} \mathrm{~d}^{-1}$ (início) a $0,020 \mathrm{~g} \mathrm{~g}^{-1} \mathrm{~d}^{-1}$ (60 dias). No P87, essa variação ficou entre $0,0210,015 \mathrm{~g} \mathrm{~g}^{-1} \mathrm{~d}^{-1}$. No P75, o crescimento variou de 0,166 a $0,080 \mathrm{~g} \mathrm{~g}^{-1} \mathrm{~d}^{-1}$ (53 dias). O melhor desempenho ocorreu no P207, com variação de 0,188 (início) a $0,017 \mathrm{~g} \mathrm{~g}^{-1} \mathrm{~d}^{-1}$ (117dias).

Com o enriquecimento dos viveiros, aumentou a oferta de alimento natural para esta espécie, repercutindo na taxa de crescimento intrínseco que mostrou uma pequena recuperação ao final do período de criação. Esse crescimento atípico da curimatã condiz com o trabalho realizado por Nuñer (1991), no qual a Prochilodus scrofa apresentou o melhor fator de condição no ambiente medianamente enriquecido. Essa condição ambiental enriquecida foi caracterizada pela elevação dos valores de condutividade elétrica, alcalinidade e clorofila-a. Woynarovich (1985) ressaltou a importância da curimatã no policultivo através do aproveitamento da matéria orgânica descartada na coluna d'água que se deposita no sedimento ou nas superfícies de pedras e macrófitas aquáticas.

Em policultivo com tambaqui (P87), o crescimento da carpa comum variou de 0,388 (início) a $0,011 \mathrm{~g} \mathrm{~g}^{-1} \mathrm{~d}^{-1}(60$ dias) e 0,361 a $0,010 \mathrm{~g} \mathrm{~g}^{-1} \mathrm{~d}^{-1}$ associado a tilápia, durante os 75 dias iniciais de criação (P78). Nos P75 e P207 a carpa apresentou bom desempenho, com crescimento variando de 0,538 a $0,0145 \mathrm{~g} \mathrm{~g}^{-1} \mathrm{~d}^{-1}$ (Figura 1).

A moderada biomassa inicial do policultivo P207 proporcionou um maior aporte de matéria orgânica no fundo dos viveiros aumentando a oferta de alimento natural para carpa comum (detritívora) e para curimatã pacu (iliófaga). $\mathrm{O}$ bom desempenho da carpa comum, expresso pelo aumento da biomassa total nos policultivos analisados, também foi observado em outros policultivos (DORIA \& LEONHARDT, 1993; SÁ, 1989).

A tilápia apresentou produtividade de $721 \mathrm{~kg} \mathrm{ha}^{-1} \mathrm{ano}^{-1}$ no policultivo com carpa e curimatã (P78). Porém, nos P75 e P207 essa produtividade foi reduzida em $70 \%$ (Figura 1 e Tabela 2). $\mathrm{O}$ crescimento $(\mathrm{p}<0,05)$ e a produtividade da tilápia ficaram aquém do previsto nesses policultivos devido ao gasto da energia (alimentar) no processo de reprodução em detrimento ao crescimento. Schreiber et al. (1998), e Stickney (2000), explicaram o baixo desempenho no crescimento de fêmeas durante o processo reprodutivo decorre da incubação oral das larvas, quando as fêmeas ficam impossibilitadas de se alimentarem. Após esse período, as desovas ocorrem a cada duas semanas, repetindo o processo reprodutivo. Verani (1980) ressaltou que a alta prolificidade da tilápia gera aumento populacional, com redução no alimento disponível, acarretando a produção de lotes heterogêneos. Desse modo, torna-se imprescindível a criação de tilápias monossexadas.

A produtividade da curimatã em policultivo com tilápia (P78) foi inferior a obtida com o tambaqui, P87 (Figura 1 e Tabela 2). Os viveiros do P78 apresentaram condições adversas ao crescimento da curimatã, evidenciando uma provável competição alimentar entre a tilápia e a curimatã. Essa competição tornou-se evidenciada no início e ao final 
do experimento, quando a utilização de recursos alimentares esteve limitada. A tilápia, devido à plasticidade na utilização desses recursos, explorou o nicho trófico da curimatã, perífiton, camada nefolóide e detritos (LOWEMCCONNELL, 1982; POPMA \& LOVSHIN, 1994; STARLING et al., 2000). Milstein \& Svirsky (1996) verificaram que a tilápia aumenta a participação de detritos na sua dieta, principalmente, os oriundos do fundo dos viveiros quando o alimento natural está exaurido.

A produtividade da carpa comum foi superior a de outros peixes (Figura 1 e Tabela 2). Verificou-se uma correlação positiva $\left(\mathrm{r}^{2}=0,881\right)$ entre a biomassa inicial e essa produtividade (Figura 2), atingindo o valor máximo de $1.606 \mathrm{~kg} \mathrm{ha}^{-1} \mathrm{ano}^{-1}$ no policultivo com tambaqui e curimatã (P87). Porém, recomenda-se a utilização de uma baixa biomassa inicial de carpa comum (P75 e P207) em policultivos considerando o multiuso dos açudes. A bioturvação causada por essa espécie, aumenta a turbidez, diminui a zona eufótica e, conseqüentemente, reduz a concentração de oxigênio dissolvido nos viveiros (NUNES, 2000).

A produtividade máxima global por espécie nos policultivos, excetuando-se a carpa comum e a tilápia (P78), ficou em $285 \mathrm{~kg} \mathrm{ha}^{-1} \mathrm{ano}^{-1}$ para a biomassa inicial de $42,7 \mathrm{~kg}$ $\mathrm{ha}^{-1}$ (Figura 2). A produtividade do tambaqui foi superior no policultivo com baixa biomassa inicial (P75) do que no com moderada biomassa (P207). Nos últimos trinta dias de criação, as taxas de crescimento intrínseco dos peixes foram extremamente baixas e alguns peixes chegaram a perder peso (P78 e P87).

A produção obtida em 90 dias pode ser extrapolada para efeito de comparação, gerando uma produtividade de $2.320 \mathrm{~kg} \mathrm{ha}^{-1} \mathrm{ano}^{-1}$. Apesar dos peixes não terem recebido alimentação suplementar, essa produtividade pode ser comparada à criação semi-intensiva. Delincé (1992) citou produtividades de 2.000 a $4.000 \mathrm{~kg} \mathrm{ha}^{-1} \cdot \mathrm{ano}^{-1}$ em criações semi-intensivas na África Central, onde foram feitas fertilizações freqüentes e os peixes receberam alimentação complementar. Milstein (1997) referiu-se à produtividade de 2700-2800 kg ha-1, em sistemas de 'acadja', na Costa do Marfim onde se utiliza bambu para desenvolver o perífiton e os peixes recebem farelo de arroz, como alimento suplementar.

A biomassa inicial de $1,0 \mathrm{~kg} \mathrm{ha}^{-1}$ de tucunaré permitiu o recrutamento de alevinos de tilápia com produção de 12 $\pm 8 \mathrm{~kg} \mathrm{ha}^{-1}$ (P75) e $28 \pm 36 \mathrm{~kg} \mathrm{ha}^{-1}$ (P207). Os elevados desvios desses dados estão associados à entrada acidental de duas traíras Hoplias sp. (P75) e uma piranha Serrassalmus sp. (P207) que também exerceram controle nas desovas. A adição acidental de outros peixes carnívoros ao policultivo ajudou no controle das desovas, corroborando os resultados obtidos por Paiva et al. (1992), em que presença de dois predadores possibilitou uma maior produção pesqueira nos grandes açudes nordestinos.

O tucunaré é um predador visual utilizado com sucesso no controle do recrutamento de tilápia. Verani (1980) determinou que as proporções de 6:1 e de 9:1 de tilápia e tucunaré, respectivamente, são eficazes no controle do recrutamento com uma maior participação de peixes de tamanho comercial ao final da criação. TeichertCoddington \& Green (1997) verificaram que o Cichlasoma managuense mostrou-se eficaz em controlar as desovas de tilápia quando estocado por área do viveiro. O melhor controle foi obtido quando utilizaram 750 C. managuense por hectare, correspondendo a $3 \%$ da população de tilápia estocada à densidade de 2,25 peixes $\mathrm{m}^{-2}$.
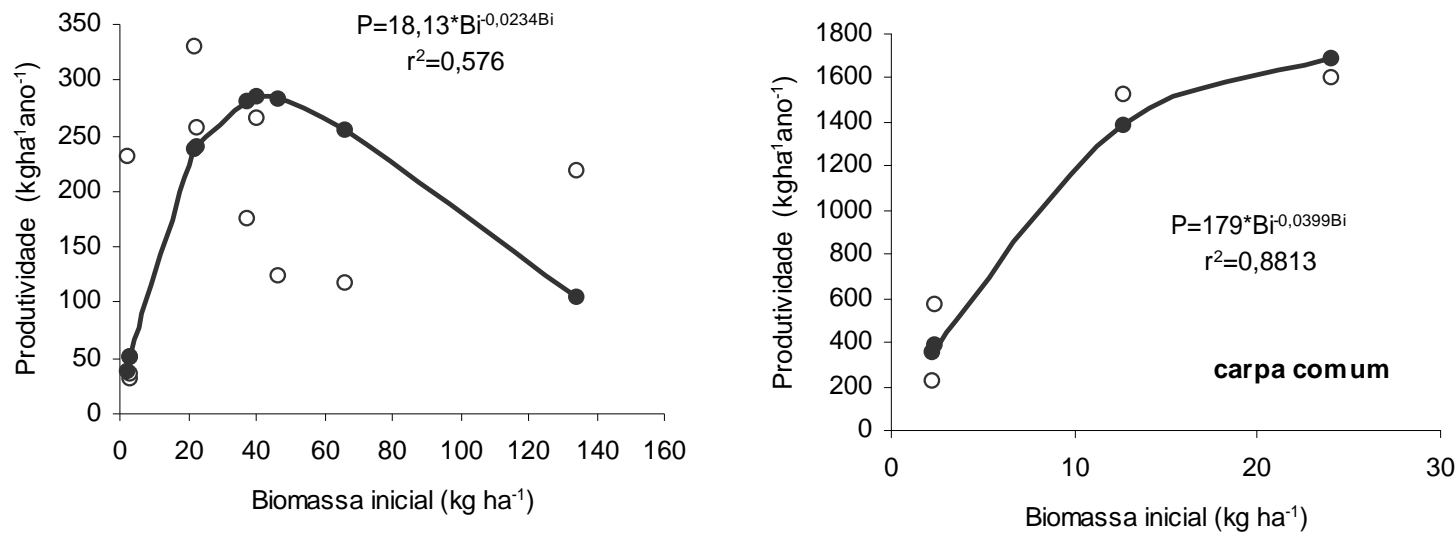

FIGURA 2 - Relação entre a biomassa inicial e a produtividade por espécie criada em policultivo. (a) Todas as espécies exceto a carpa comum e a tilápia (P78).

Ciênc. agrotec., Lavras, v. 30, n. 6, p. 1083-1090, nov./dez., 2006 


\section{CONCLUSÕES}

A biomassa inicial de $75 \mathrm{~kg} \mathrm{ha}^{-1}(60: 30: 4: 3: 3 \%$ de tilápia, tambaqui, carpa, curimatã e tucunaré, respectivamente) proporcionou o melhor crescimento e produtividade de peixes.

$\mathrm{O}$ alimento natural foi limitante ao tambaqui a partir do segundo mês de criação.

\section{AGRADECIMENTOS}

À Coordenação de Aperfeiçoamento de Pessoal do Ensino Superior - CAPES, à Fundação de Amparo à Ciência e Tecnologia de Pernambuco - FACEPE através da APQ120-506/97. À 'Sun West Aquafarms' e a Unidade de Piscicultura de Bebedouro da CODEVASF pela disponibilização da infra-estrutura. Às estações de piscicultura: Paulo Viegas (IBAMA), Johei Koike (UFRPE), Paulo Afonso (CHESF), Oceano Atlântico Linhares (DNOCS) e a Divisão de Recursos Pesqueiros (SPRA-PE) pelo fornecimento de alevinos.

\section{REFERÊNCIAS BIBLIOGRÁFICAS}

DELINCÉ, G. The ecology of fish pond ecosystem: with special reference to Africa. Netherlands: Kluwer, 1992. 230 p.

DORIA, C. R. C.; LEONHARDT, J. H. Análise do crescimento de Cyprinus carpio (Pisces: Cyprinidae) em sistema de policultivo semi-intensivo com arraçoamento e adubação orgânica. Revista UNIMAR, Marília, v. 15, p. 223-231, 1993.

LIMA, C. A.; GOULDING, M. Os frutos do tambaqui: ecologia, conservação e cultivo na Amazônia. Tefé: Sociedade Civil Mamirauá/MTC-CNPq, 1998. 186 p.

LOWE-McCONNELL, R. H. Tilápias in fish communities. In: PULLIN, R. S. V.; LOWE-McCONNELL, R. H. (Eds.). The biology and culture of tilapias. Manila: ICLARM, 1982. p. 83-113.

MILSTEIN, A. Fish species interactions. Prague: EIFAC/ FAO/SYMP.R.5, 1990.

MILSTEIN, A. Do management procedures affect the ecology of warm water polyculture ponds? Word Aquaculture, Baton Rouge, v. 28, n. 3, p. 12-19, 1997.
MILSTEIN, A.; SVIRSKY, F. Effect of fish species combinations on water chemistry and plankton composition in earthen fish ponds. Aquaculture Research, [S.1.], v. 27, p. 79-90, 1996.

MOLLE, F.; CADIER, E. Manual do pequeno açude: construir, conservar e aproveitar pequenos açudes no Nordeste brasileiro. Recife: SUDENE/ORSTON, 1992. 521 p.

MONCAYO, T. L.; HERNANDEZ, C. S. Utilización de alimentos de complemento en el cultivo semi-intensivo de alevinos de Colossoma macropomum. In: SIMPÓSIO LATINO AMERICANO DE AQUICULTURA, 6.; SIMPÓSIO BRASILEIRO DE AQUICULTURA, 5., 1988, Florianópolis. Anais... Florianópolis: ALA/ABRAq, 1988. p. 520-525.

NUÑER, A. P. O. Estudo interativo entre as variáveis ambientais e o desenvolvimento do curimbatá,Prochilodus scrofa (Steindachner, 1881), em ambiente confinado. 1991. 183 f. Dissertação (Mestrado) - Universidade Federal de São Carlos, São Carlos, 1991.

NUNES, Z. M. P. Interações entre peixes onívoros: proposta de um policultivo para pequenos açudes do semiárido nordestino. 2000. 256 f. Tese (Doutorado) Universidade Federal de São Carlos, São Carlos, 2000.

PAIVA, M. P.; PETRERE JUNIOR, M.; PETENATE, A. J.; NEPOMUCENO, F. H.; VASCONCELOS, E. A. Relationship between the number of predatory fish species and fish yield in large Northeastern Brazilian reservoirs. Rehabilitation of Freshwater Fisheries, [S.1.], p. 120-129, 1992.

POPMA, T. J.; LOVSHIN, L. L. Worldwide prospects for commercial production of tilápia. Auburny: ICLARM; Auburny University, 1994. 40 p.

SÁ, M. F. P. de. Efeito da adubação orgânica sobre o crescimento de Cyprinus carpio, Prochilodus cearensis e Colossoma macropomum em experimento de policultivo. 1989. 162 f. Dissertação (Mestrado) - Universidade Federal de São Carlos, São Carlos, 1989.

SCHREIBER, S.; FOCKEN, U.; BECKER, K. Individually reared female Nile tilapia (Oreochromis niloticus) can grow faster than males. Journal of Applied Ichthyology, [S.l.], v. 14, p. 43-47, 1998. 
STARLING, F.; LAZZARO, X.; WALTER, T.; RIBEIRO, M. Large-scale extensive tilapia production in a tropical eutrophic reservoir (Lago Paranoá, Brasília, Barasil): environmental and socio-economical benefits from biomanipulation. In: INTERNATIONAL SYMPOSIUM ON TILAPIA AQUACULTURE, 5., 2000, Rio de Janeiro. Proceedings... Rio de Janeiro: [s.n.], 2000. p. 631.

STICKNEY, R. R. Status of research on tilapia. In: COSTAPIERCE, B. A.; RAKOCY, J. E. (Eds.). Tilapia aquaculture in the Americas 2. Baton Rouge WAS, 2000. p. 21-33.

TEICHERT-CODDINGTON, D. R.; GREEN, B. W. Experimental and commercial culture of tilapia in Honduras.
In: COSTA-PIERCE, B. A.; RAKOCY, J. E. (Eds.). Tilapia Aquaculture in the Americas 1. Baton Rouge: Aquaculture Society, 1997. p. 142-162.

VERANI, J. R. Controle populacional em cultivo intensivo consorciado entre a tilápia do Nilo Sarotherodon niloticus (Linnaeus, 1757) e o tucunaré comum, Cichla ocellaris Schneider, 1801 aspectos quantitativos. 1980. $116 \mathrm{f}$. Dissertação (Mestrado) - Universidade Federal de São Carlos, São Carlos, 1980.

WOYNAROVICH, E. Manual de piscicultura. Brasília, DF: CODEVASF/MINTER, $1985.71 \mathrm{p}$. 